\title{
PICOSECOND LASER STUDIES ON THE EFFECT OF STRUCTURE AND ENVIRONMENT ON INTERSYSTEM CROSSING IN AROMATIC CARBENES
}

\author{
J.G. LANGAN, E.V. SITZMANN and K.B. EISENTHAL \\ Department of Chemistry, Columbin University, New York, New York 10027, USA
}

Received 26 July 1984

\begin{abstract}
A large solvent polarity effect on the rate of singlet to triplet intersystem crossing ( $k_{\mathrm{ST}}$ ) has been observed in the carbenes, diphenylcarbene (DPC) and dicycloheptadienylidene (DCHD). It is found that both $k$ ST and the energy splitting $\left(\Delta E_{\mathrm{ST}}\right)$ separtaing the singlet and triplet states decrease as the solvent polarity increases for the aromatic carbenes. This "inverse" gap effect, i.e. the time for intersystem crossing decreases with increasing energy gap, is explained by an offresonance intersystem crossing from the singlet to a sparse triplet vibronic manifold characteristic of a small energy gap. The trend in $\Delta E_{\mathrm{ST}}$, which is proposed to be responsible for the variation in $k_{\mathrm{ST}}$ for DPC, DCHD and structurally related aromatic carbenes, is suggested to arise from the variation in the bond angle of the central methylene carbon atom.
\end{abstract}

\section{Introduction}

Of crucial importance in determining the spectroscopy, dynamics of energy relaxation and chemistry of carbenes are its two lowest electronic states $[1-4]$. The nature of these electronic states is dependent on the groups $R_{1}$ and $R_{2}$ attached to the central carbon. For aromatic carbenes it is generally found that they have a triplet ground state [5-7] with a closely neighboring singlet state. The occupancy of the non-bonding molecular orbitals of the carbene determines the multiplicity of the state as shown in scheme 1.

The intramolecular dynamics of aromatic carbenes, the subject of this paper, is dominated by intersystem crossing between the lowest singlet and ground triplet

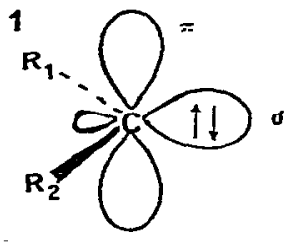

SINGLET

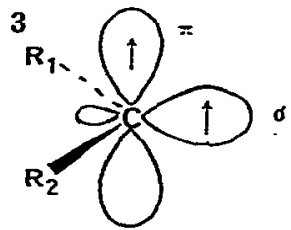

TRIPLET
Scheme 1. Electronic configurations of lowest singlet $(\sigma)^{2}$ and triplet $(\sigma)^{1}(\pi)^{1}$ states of carbene.

0 009-2614/84/\$ 03.00 @ Elsevier Science Publishers B.V. (North-Holland Physics Publishing Division) states. In the solvent acetonitrile at room temperature we found for diphenylcarbene (DPC) that the intersystem crossing time, $k_{\mathrm{ST}}^{-1}$, from the singlet to the triplet was 310 ps and the reverse triplet to singlet crossing time, $k_{\mathrm{TS}}^{-1}$, was $90 \mathrm{~ns}$ [4]. On obtaining these kinetic results for DPC in acetonitrile, one would like to know how applicable these findings are to other solvent environments, i.e. to what extend does the solvent affect the intramolecular spin conversion dynamics of the carbene? Prior to this work it was generally assumed that the solvent had no effect on the carbene spin conversion rates. A further aspect of this study seeks to address the issue of the relation of the carbene structure to the dynamics of intersystem crossing. To this end the singlet to triplet relaxation in the aromatic carbene dicycloheptadienylidene (DCHD) was studied. DCHD is akin to DPC in having two phenyl groups but differs in having them restricted by a connecting ethylene bridge.

The approach used in the measurement of intersystem crossing was laser-induced fluorescence following<smiles>c1ccc(Cc2ccccc2)cc1</smiles>

DPQC<smiles>c1ccc2c(c1)CCc1ccccc1C2</smiles>

DCED 
Generation step

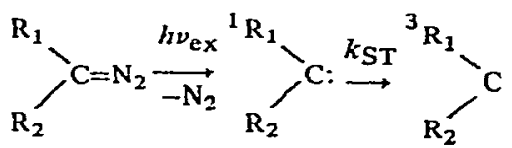

Detection step (laser induced fluorescence)

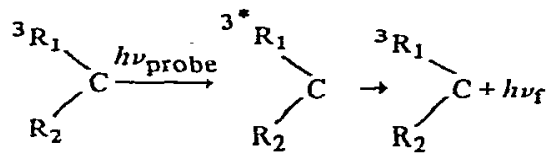

Scheme 2. Laser-induced fluorescence technique to measure growth kinetics of ground triplet carbene.

photodissociation of the carbene precursor, given by scheme 2.

\section{Experimental}

The laser-induced fluorescence experiment has been described previously [8]. Briefly the output of a passively mode-locked Nd/YAG laser (1064 nm, 25-30 ps fwhm) was frequency tripled $(355 \mathrm{~nm})$ and quadrupled $(266 \mathrm{~nm})$ to produce the probe and excitation pulses respectively. The carbene is generated in the singlet state by excitation of the diazo compound at $266 \mathrm{~nm}$. The formation of the carbene in its ground triplet state was monitored by laser-induced fluorescence using a weak probe pulse (266 nm for DPC and 355 for DCHD) at various time delays. All solutions were flowed under a nitrogen atmosphere, at $298 \mathrm{~K}$. Rate constants were determined by minimizing the standard deviation between the data acquired and a single exponential rise convoluted with the pump and probe laser pulse widths.

Diphenyldiazomethane was prepared by basecatalyzed oxidation of the hydrazone using $\mathrm{HgO}$ [9]. Diazodibenzocycloheptadienylidene was prepared from the corresponding tosylhydrazone [7]. Both diazo compounds were subjected to several recrystallyzations from pentane until satisfactory melting points were obtained. All solvents were freshly distilled under nitrogen before use, except 3-methylpentane which was purified by passage over neutral $\mathrm{Al}_{2} \mathrm{O}_{3}$.

\section{Results and discussion}

\subsection{Solvent effect}

To explore the effects of solvent perturbations on intersystem crossing the singlet to triplet rate constant $k_{\mathrm{ST}}$ of DPC was measured in a series of solvents. As shown in fig. 1 the intersystem crossing rate depends strongly on the choice of solvent [10]. In isooctane, a non-polar hydrocarbon, the time of intersystem crossing, $k_{\mathrm{ST}}^{-1}$, was found to be $95 \mathrm{ps}$, a factor of three faster than in acetonitrile $(310 \mathrm{ps})$. For the wide range of solvents used, it turned out that there was a clear ordering between the rate of singlet to triplet conversion and the polarity of the solvent, the the rate being larger in the less polar solvents. It is found that $\log \left(k_{\mathrm{ST}}\right)$ sacles linearly with solvent polarity as measured by the solvent polarity parameter $E_{\mathrm{T}}(30)[11]$. No correlation was obtained with other solvent parameters which could be related to possible carbene-solvent complexes or parameters such as viscosity which could influence the dynamics of the structural change in the transition from the singlet to the triplet. The variation of $k_{\mathrm{ST}}$ over a limited temperature range is also shown in fig. 1 . If the origin of this dependence is a barrier the height would be roughly $600 \mathrm{~cm}^{-1}$. However, we recognize that the solvent

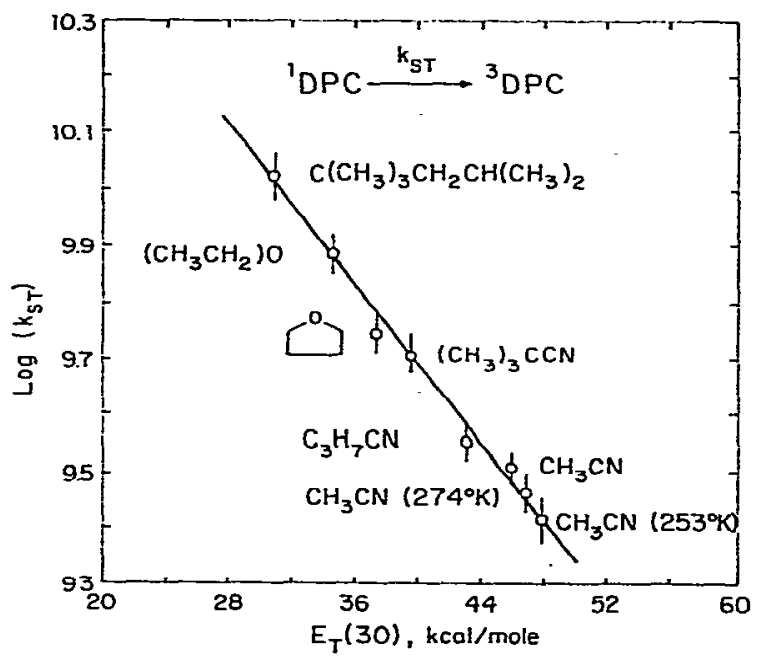

Fig. 1. Intersystem crossing rate of DPC as a function of the solvent polarity parameter $E_{\mathrm{T}}(30)$. 
polarity increases with decreasing temperature. Thus, the temperature effect could be due to the temperature-dependent polarity altering $k_{\mathrm{ST}}$ rather than the presence of an activated process. We measured the $E_{\mathrm{T}}(30)$ over the same range of temperature and calculated how $k_{\mathrm{ST}}$ would change, the solid line is the calculated curve. We conclude from the agreement shown that the temperature dependence can be explained by the temperature effect on the solvent polarity, and therefore that there is no barrier for singlet to triplet crossing or at most a very small one.

\subsection{Effect of structure}

To determine if the marked solvent polarity effect on singlet to triplet energy relaxation was peculiar to diphenylcarbene we studied another aromatic carbene, dibenzocycloheptadienylidene (DCHD). This molecule is similar to DPC, differing by an ethylenic bridge which connects the two phenyl rings to the central carbon atom. Onc effect of the restricting $-\mathrm{CH}_{2}-$ groups in DCHD is to increase the angle between the central carbon and the connected phenyl rings relative to DPC. Results of the laser-induced fluorescence experiment for DCHD are shown in fig. 2. From our

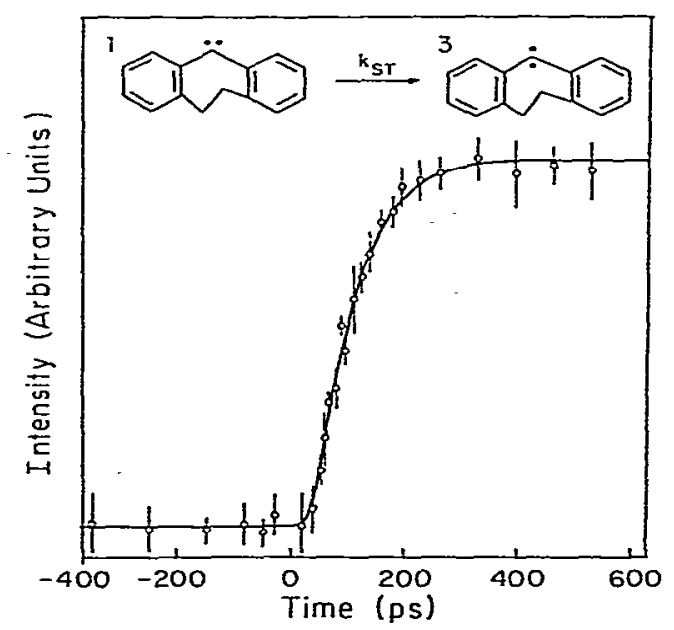

Fig. 2. Laser-induced fluorescence of DCHD in tetrahydrofuran at $298 \mathrm{~K}$ as a function of time delay between $266 \mathrm{~nm}$ excitation and $355 \mathrm{~nm}$ probe pulses. The solid curve is a calculated fit.

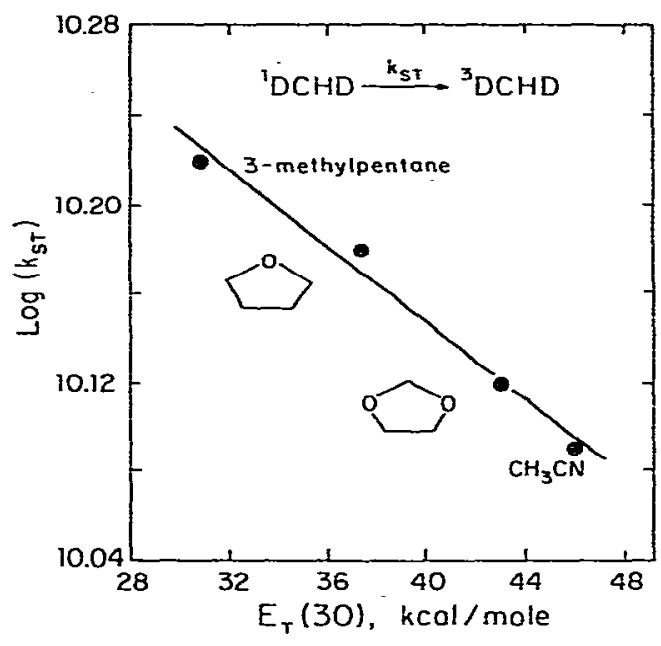

Fig. 3. Intersystem crossing rate of DCHD as a function of the solvent polarity parameter $E_{\mathrm{T}}(30)$.

measurements of $k_{\mathrm{ST}}$ in a variety of solvents we find that DCHD also manifests an exponential dependence of $k_{\mathrm{ST}}$ on the solvent polarity parameter $E_{\mathrm{T}}(30)$, hence giving the linear relationship shown in fig. 3 . The slopes for DPC and DCHD differ but the same exponential dependence of $k_{\mathrm{ST}}$ on $E_{\mathrm{T}}(30)$ is seen. Related to these observations is a recent and most interesting report on another carbene which is in agreement with the solvent trend reported here. Fluorenylidene in solution at room temperature was observed to have a faster intersystem crossing in the non-polar solvent cyclohexane than in the polar solvent acetonitrile [12]. From these various studies we can conclude that the solvent does indeed play a key role in singlet-triplet relaxation in aromatic carbenes, due to the sharp differences in the polarities of the lowest singlet and triplet states.

To describe the effects of solvent polarity on spin conversion in carbenes consider the electronic nature of the singlet and triplet states. The singlet state, with both non-bonding electrons localized in the same $(\sigma)^{2}$ orbital of the central carbon, will be significantly more polar than the triplet state $(\sigma)^{1}(\pi)^{1}[10,13]$ (scheme 1). It is, therefore, to be expected that the polar singlet will be strongly stabilized, i.e. lowered in energy in the more polar solvents, whereas the less polar triplet will experience less stabilization. This 
differential stabilization between the singlet and triplet would, therefore, result in the singlet-triplet energy gap decreasing as the solvent polarity increases. This prediction that the singlet-triplet energy gap is larger in the non-polar than in the polar solvent was confirmed experimentally in DPC. We found from measurements of DPC in non-polar isooctane that the energy gap is $1400 \mathrm{~cm}^{-1}$ compared with $950 \mathrm{~cm}^{-1}$ for polar acetonitrile [4].

The issue now becomes why intersystem crossing, which involves the conversion of electronic energy of the singlet into vibrational energy of the triplet, increases in rate as the energy gap increases. This is a seemingly surprising result in that intersystem crossing unusually decreases as the gap increases, at least for large gaps. The key factor may be that the aromatic carbenes are in a small energy gap domain. The consequence of the small energy gap is that there is a sparse manifold of triplet vibronic states (ground triplet plus vibrations) with which the singlet is effectively couplet [14]. The appropriate description is an offresonance coupling between $S_{1}$ and a number of triplet vibronic levels [15], as given by

$k_{\mathrm{ST}} \propto\left\langle\sum_{\mathrm{i}} \frac{\left|\left(H_{\mathrm{SO}}\right)_{\mathrm{S}, \mathrm{T}_{\mathrm{f}}}\right|^{2} \Gamma_{\mathrm{T}_{\mathrm{f}}}}{\left(E_{\mathrm{S}}-E_{\mathrm{T}_{\mathrm{f}}}\right)^{2}+\left(\Gamma_{\mathrm{Tf}} / 2\right)^{2}}\right\rangle$,

where $\left(H_{\mathrm{SO}}\right)_{\mathrm{S}, \mathrm{T}_{\mathrm{f}}}$ is the spin-orbit coupling between the initial singlet state and $T_{f}$, one of the triplet vibrational states, $\Gamma_{T_{f}}$ is the width of $T_{f}$ due to its coupling with the solvent and the average is over the initial singlet state distribution. The vibrations in the triplet which provide the coupling with the singlet are in turn coupled to other vibrations in the molecule and the solvent background. The structure of these triplet vibronic levels becomes increasingly congested in the neighborhood of $S_{1}$ as the energy gap increases, due to the possible contributions of higher-frequency vibrations and due to the decrease in the spacing between the triplet vibronic energy levels as the energy gap increases. Accordingly the rate of $S_{1} \rightarrow T_{0}$ intersystem crossing increases.

This physical picture for the inverse energy gap ef fect is consistent with observations of singlet to triplet intersystem crossing in three different aromatic carbenes. They are diphenylcarbene, dicycloheptadienylidene and fluorenylidene. In each case it is found that $k_{\mathrm{ST}}$ is largest in those solvents for which the carbene has the largest singlet-triplet energy gap. This description is not anticipated to be applicable when the energy gap increases beyond a few thousand $\mathrm{cm}^{-1}$. We would expect that as the gap becomes larger the increasing density of vibronic states in the triplet manifold which couple to $S_{1}$ will lead to a declining importance of the inverse gap effect. When the energy gap becomes large we anticipate approaching the usual energy gap effect found in the statistical limit, i.e. the rate of $S \rightarrow T$ intersystem crossing decreases with increaseing energy gap due to poorer Franck-Condon factors [14].

Comparing the intersystem crossing rate $k_{\mathrm{ST}}$ for the three aromatic carbenes for which there is data we can consider the effects of carbene structure on the spin conversion process. The values of $k_{\mathrm{ST}}$ in a given solvent are in the order $k_{\mathrm{ST}}(\mathrm{DCHD})>k_{\mathrm{ST}}(\mathrm{DPC})$ $>k_{\mathrm{ST}}(\mathrm{FL})$. The same ordering is found in the magnitude of the energy gaps $\Delta E_{\mathrm{ST}}$ (DCHD, $\left.\approx 1700 \mathrm{~cm}^{-1}[16]\right)>\Delta E_{\mathrm{ST}}\left(\mathrm{DPC}, \approx 950 \mathrm{~cm}^{-1}[4]\right)$ $>\Delta E_{\mathrm{ST}}\left(\mathrm{FL}, \approx 600 \mathrm{~cm}^{-1}\right.$ [12]). The origin of the ordering of the $k_{\mathrm{ST}}$ values could be simply due to the differences in the spin-orbit matrix elements in the three molecules. However, it is tempting to speculate that the major factor is the energy gap, i.e. the larger the energy gap the faster is the intersystem crossing $k_{\mathrm{ST}}$. As $\Delta E_{\mathrm{ST}}$ increases the greater congestion of triplet vibronic states that are couplet to $S_{1}$ results in larger $k_{\mathrm{ST}}$ values. This is the same physical picture used to explain the solvent effect on the $k_{\mathrm{ST}}$ values for each of these carbenes.

To understand the origin of the variation of $\Delta E_{\mathrm{ST}}$ and thus that of $k_{\mathrm{ST}}$ we consider how the different geometrical structures could alter $\Delta E_{\mathrm{ST}}$. Theoretical calculations on DPC indicate that the singlet-triplet splitting decreases as the angle defined by the two bonds to the central carbon decreases [17]. Qualitatively as the molecule goes from a linear towards a bent geometry, i.e. the central angle decreases, the percent 2 s character in the orbital increases thereby lowering the orbital energy. In addition it is found that the singlet $(\sigma)^{2}$ is more stabilized than the triplet $(\sigma)^{1}(\pi)^{1}$ leading to a decrease in energy gap as the angle decreases [17]. The evidence that supports an increase in the central angle in the series DCHD > DPC $>$ FL is obtained from EPR data [18]. The value of the zero-field splitting parameter $|E| / h c$, which is a measure of the deviation of the spin-spin inter- 


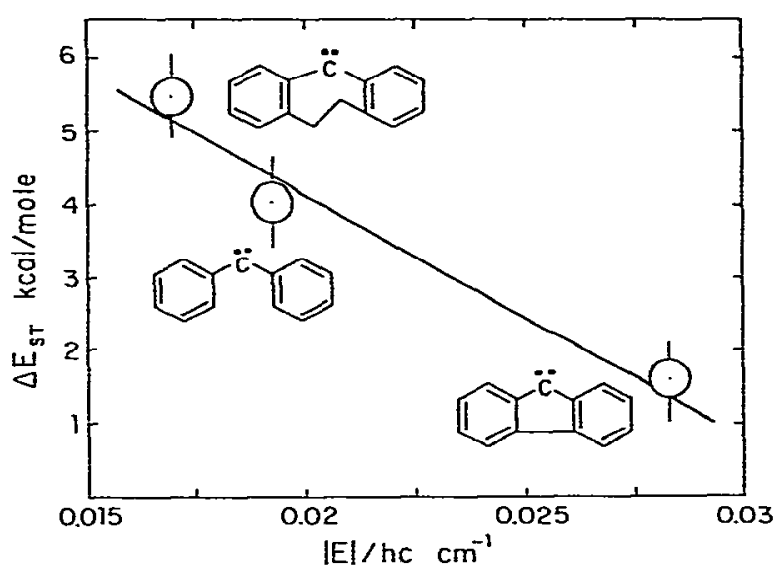

Fig. 4. PJot of $\Delta E_{\mathrm{ST}}$ versus $[E] / h c$ various carbenes.

action from cylindrical symmetry in the spin dipoledipole Hamiltonian, is an indication of the $2 \mathrm{~s}$ character in the orbital. Crude estimates of $2 \mathrm{~s}$ character can also be obtained from the ratio of the $|E| /|D|$ values where $|D| / h c$ is proportional to the separation of the unpaired spins. It has been previously noted that $|E| / h c$ and $|E| /|D|$ decrease as the bond angle increases and is therefore a measure of the decrease in the percent $2 \mathrm{~s}$ character in the non-bonding $\sigma$ orbital $[18,19]$. As shown in fig. $4, \Delta E_{\mathrm{ST}}$ increases as $|E| / h c$ decreases, showing that an increase in bond angle could be the structural change responsible for the change in $\Delta E_{\mathrm{ST}}$.

Another carbene that is important to consider in the present context is 9-mesityl-9,10-dihydro-9boranthrylidene (BA). Measurements of the $|D| / h c$ and $|E| / h c$ values $\left(0.360 \mathrm{~cm}^{-1}\right.$ and $0.0156 \mathrm{~cm}^{-1}$, respectively) for $B A$ [20] are similar to DCHD, implying similar bond angles for the two carbenes. If bond angle is a critical parameter determining the energetics and spin conversion rates of the carbene, as we have suggested, then both BA and DCHD should have roughly similar values for $\Delta E_{\mathrm{ST}}$ and $k_{\mathrm{ST}}$. This indeed appears to be the case. Studied have yielded an upper limit on intersystem crossing given as $k_{\mathrm{ST}}$ of $>1 \times$ $10^{10} \mathrm{~s}^{-1}$ [20] (the same as DCHD, table1), and an estimate of $\geqslant 5.2 \mathrm{kcal} / \mathrm{mole}$ for $\Delta E_{\mathrm{ST}}[20]$, also similar to DCHD. BA thus fits squarely within the framework of a strong dependence of the energy gap and corresponding relaxation rates between the singlet and triplet states to the carbene bond angle.<smiles>Cc1cc(I)cc(C)c1B1c2ccccc2[CH]c2ccccc21</smiles><smiles>Cc1cc(C)c(C#Cc2c(C)cc(C)cc2C)c(C)c1</smiles>

Additional evidence that the energy gap increases as the carbene assumes a more linear geonetry can be offered by way of considering the difference in the spin-state-dependent chemistry observed for the various aromatic carbenes. For example comparison of the reaction rates of DCHD, DPC and FL with a number of different carbene quenchers has provided evidence that both DCHD and DPC have a larger energy gap than FL [21]. Finally, dimesitylcarbene (DMC) is another example of an aromatic carbene where the bond angle is proposed to be of key importance in determining its energetics. It has been found [22] that $D M C$ has an exceedingly small $|E| / h c$ value $(0.0082$ $\mathrm{cm}^{-1}$ ) which again argues for a more linear structure. A more linear geometry for DMC is reasonable if one considers the steric repulsions between the mesityl groups bonded to the central methylene carbon. The reactions of DMC are shown to occur predominantly from the ground triplet state suggesting that the higher lying singlet state may not be thermally accessible, i.e. it has a large $\Delta E_{\mathrm{ST}}$. Interestingly, if one were to extend the linear relationship between $|E| / h c$ and $\Delta E_{\mathrm{ST}}$ (as given by fig. 4 ) down to the low $[E \mid / h c$ value observed for DMC, then one would expect DMC to have

Table 1

Intersystem crossing time of DCHD in various solvents at $298 \mathrm{~K}$

\begin{tabular}{lll}
\hline Solvent & $\begin{array}{l}10^{-10} k_{\mathrm{ST}} \\
\left(\mathrm{s}^{-1}\right)\end{array}$ & $\begin{array}{l}E_{\mathrm{T}}(30)^{\mathrm{a})} \\
(\mathrm{kcal} / \mathrm{mole})\end{array}$ \\
\hline 3-methylpentane & $1.66 \pm 0.06$ & 30.9 \\
$\begin{array}{l}\text { tetrahydrofuran } \\
\text { 1,3-dioxolane }\end{array}$ & $1.54 \pm 0.05$ & 37.9 \\
acetonitrile & $1.33 \pm 0.06$ & 43.1 \\
\hline
\end{tabular}

a) See ref. [11]. 
an energy gap of $\approx 8-9 \mathrm{kcal} / \mathrm{mole}$, which would be entirely consistent with its reported chemistry [22].

\section{Conclusions}

Using picosecond laser techniques we have observed a large solvent effect on the intersystem crossing rate constant $k_{\mathrm{ST}}$ in the aromatic carbenes, diphenylcarbene (DPC) and dicycloheptadienylidene (DCHD). The solvent polarity is the dominant feature responsible for changes in $k_{\mathrm{ST}}$. It is found that the singlet-triplet energy gap changes with polarity and thereby alters the intersystem crossing dynamics. Increasing polarity decreases the gap which leads to a slower rate constant $k_{\mathrm{ST}}$. This "inverse" gap effect, i.e. the time for intersystem crossing decreasing with increasing energy gap, is explained by an off-resonance intersystem crossing from the singlet to a sparse triplet vibronic manifold characteristic of a small energy gap. In comparing the intersystem crossing rate constants of the three different aromatic carbenes DPC, DCHD and FL in a common solvent we find that the intersystem crossing time scales inversely with the singlet-triplet energy gap. This is the same trend of $k_{\mathrm{ST}}$ with the solventinduced changes in energy gap we have already noted for each of the carbenes. This trend in $\Delta E_{\mathrm{ST}}$ values, which is proposed to be responsible for the variation in intersystem crossing $\left(k_{\mathrm{ST}}\right)$ as well as chemical reaction rates, for a series of structurally related aromatic carbenes is speculated to arise from the variation in the central carbon to ligands angle.

\section{Acknowledgement}

We wish to thank the National Science Foundation, the Air Force Office of Scientific Research, and the Joint Services Electronic Program 29-82-K-0080 for their generous support of this work.

\section{References}

[1] W. Kirmse, Carbene chemistry, 2nd Ed. (Academic Press, New York, 1971);

R.A. Moss and M. Jones Jr., eds., Carbenes, Vols. 1 and 2 (Wilcy-Interscience, New York, 1975);

H. Dürr, Topies Current Chem. 55 (1975) 87;

A.M. Trozzolo, Accounts Chem. Res. 1 (1968) 329.
[2] G.L. Closs and B.E. Rainbow, J. Am. Chem. Soc. 98 (1976) 8190;

D. Bethel, D. Whittaker and J.D. Callister, J. Chem. Soc. (1965) 2466;

D. Bethel, G. Stevens and P. Tickel, Chem. Commun. (1970) 792;

P.P. Gasper, B.L. Whitsel, M. Jones Jr. and J.B. Lambert, J. Am. Chem. Soc. 102 (1980) 6108;

D. Griller, L. Hadel, A.S. Nazran, M.S. Platz, P.C. Wong, J.G. Savino and J.C. Scaiano, J. Am. Chem. Soc. 106 (1984) 2?27;

P.D. Gasper, C.-T. Lin, B.L. Whitsel Dunbar, D.P. Mack and P. Balasubramanian, J. Am. Chem. Soc. 106 (1984) 2128 .

[3] K.B. Eisenthal, J.J. Turro, J. Aikawa, J.A. Butcher Jr. C. Dupuy, G. Hefferon, W.M. Hetherington III, G.M. Korenowski and M. McAuliffe, J. Am. Chem. Soc. 102 (1980) 6563.

[4] K.B. Eisenthal, N.J. Turro, E.V. Sitzmann, I.R. Gould, G. Hefferon, J. Langan and $Y$. Cha, Tetrahedron, to be published.

[5] C.A. Hutchison and B.E. Kohler, J. Chem. Phys. 51 (1969) 3357;

R.W. Brandon, G.L. Closs, C.E. Davoust, C.A. Hutchinson, B.F. Kohler and R. Silbey, J. Chem. Phys. 43 (1965) 2006;

C.A. Hutchinson and G.A. Pearson, J. Chem. Phys. 47 (1967) 520 .

[6] I. Moritani, S.-I. Murahashi, M.H. Yamamoto, K. Itoh and N. Mataga, J. Am. Chem. Soc. 89 (1967) 1259;

I. Moritani, S.-I. Murahashī, N. Nishino, Y. Kimura and H. Tsubomura, Tetrahedron Letters (1966) 373; I. Moritani, S.-I. Murahashi, H. Ashitaka, K. Kimura and H. Tsubomura, J. Am. Chem. Soc. 90 (1968) 5918; S.-I. Murahashi, I. Moritani and M. Nishino, Tetrahedron 27 (1971) 5131.

[7] I. Moritani, S.-I. Murahashi, K. Yoshinga and H. Ashitaka, Bull. Chem. Soc. Japan 40 (1967) 1506.

18] C. Dupuy, G.M. Korenowski, M. McAuliffe, W.M. Hetherington III and K.B. Eisenthal, Chem. Phys. Letters 77 (1981) 272;

E.V. Sitzmann and K.B. Eisenthal, in: Applications of picosecond spectroscopy to chemistry, ed. K.B. Eisenthal (Reidel, Dordrecht, 1984) p. 41.

[9] J.B. Miller, J. Org. Chem. 24 (1959) 560.

[10] E.V. Sitzmann, J. Langan and K.B. Eisenthal, J. Am. Chem. Soc. 106 (1984) 1868.

[11] C-Reichardt, in: Molecular interactions, Vol. 3, eds. H. Ratajczak and W.J. Orville-Thomas (Wiley, New York, 1982) p. 241;

E.M. Kosower, J. Am. Chem. Soc. 80 (1958) 3253.

[12] P.B. Grasse, B.-E. Brauer, J.J. Zupanic, K.J. Kaufmann and G.B. Schuster, J. Am. Chem. Soc. 105 (1983) 6833.

[13] L. Salem and C. Roland, Angew. Chem. Intern. Ed. Engl. 11 (1972) 92.

[14] C.W. Robinson and R.P. Frosch, J. Chem. Phys. 37 (1962) 1962; 38 (1963) 1187;

J. Jortner, S.A. Rice and R.M. Hochstrasser, Advan. 
Photochem. 7 (1969) 149;

B.R. Heury and W. Siebrand, in: Organic molecular photophysics, Vol. 1, ed. J.B. Birks (Wiley, New York, 1973) p. 153;

K.F. Freed, Topics Appl. Phys. 15 (1976) 23.

[15] A. Nitzan and J. Jortner, Theoret. Chim. Acta 29 (1973) 97.

[16] K.B. Eisenthal, N.J. Turro, G.L. Langan, Z.Z. Ho, I.R. Gould and E.V. Sitzmann, to be published.

[17] J. Metcalfe and E.A. Halevi, J. Chem. Soc. Perkin Trans. I (1977) 634;

R. Hoffman, G.D. Zeiss and G.W. van Dine, J. Am. Chem. Soc. 90 (1968) 1485.

[18] E. Wasserman, A.M. Trozzolo and W.A. Yager, J. Chem. Phys. 40 (1964) 2408;
E. Wasserman, W.A. Yager and V.J. Kuck, Cherr. Phys. Letters 7 (1970) 409;

E. Wasserman, V.J. Kuck, R.S. Hutton and W.A. Yager, J. Am. Chem. Soc. 92 (1970) 7491.

[19] J. Higuchi, J. Chem. Phys. 39 (1963) 1339.

[20] S.C. Lapin, B.-E. Brauer and G.B. Schuster, J. Am. Chem. Soc. 106 (1984) 2092.

[21] L.M. Hadel, M.S. Platz, B.B. Wright and J.C. Scaiano, Chem. Phys. Letters 105 (1984) 539.

[22] A.S. Nazran, E.J. Gabe, Y. LePage, D.J. Northcott, J.M. Park and D. Griller, J. Am. Chem. Soc. 105 (1983) 2912;

A.S. Nazran and D. Griller, J. Chem. Soc. Chem. Commun. (1983) 850. 